\title{
Diabetes Mellitus e Suas Implicações na Osteointegração de Implantes Dentários: Revisão Sistematizada da Literatura
}

Diabetes Mellitus and Implications with the Osteointegration of Dental Implants: Systematized Review of Literature Diabetes Mellitus y Implicaciones con la Osteointegración de Implantes Dentales: Revisión Sistematizada de la Literatura Edgladisson Ramos DA SILVA

Cirurgião-Dentista pela Faculdade Uninassau, 58030-000 - João Pessoa - PB, Brasil https://orcid.org/0000-0002-7456-8630

Jales de Brito MENESES

Cirurgião-Dentista pela Faculdade Uninassau, 58030-000 - João Pessoa - PB, Brasil https://orcid.org/0000-0002-2110-8884 Herrison Félix Valeriano DA SILVA

Mestrando em Odontologia da Universidade Federal da Paraíba (UFPB) - 58051-900 João Pessoa - PB, Brasil https://orcid.org/0000-0001-6714-3151 Myllenna Nayara de França ALVES

Graduada em Odontologia, FACENE, 58067-695 João Pessoa - PB, Brasil https://orcid.org/0000-0002-7544-2730

Rafaella Bastos LEITE

Professora Doutora, Curso de Odontologia da Faculdade Uninassau, 58030-000, João Pessoa - PB, Brasil https://orcid.org/0000-0002-3304-120X

\section{Resumo}

Introdução: Pacientes com diabetes mellitus apresentam certo comprometimento no processo de cicatrização, assim como a neoformação óssea. Objetivo: Analisar por meio de uma revisão sistemática da literatura a viabilidade da reabilitação oral por meio de implantes dentários em pacientes diagnosticados com Diabetes Mellitus. Material e método: Foi realizada uma revisão sistemática de literatura com análise de artigos no banco de dados disponíveis no Google Acadêmico, Lilacs e PubMed. Utilizaram-se os descritores "Osseointegração", "Implantes Dentários" e "Diabetes Mellitus". Foram selecionados textos em qualquer idioma e a sequência de acordo com a relevância. Os critérios de inclusão usados foram artigos e livros publicados entre os anos de 2016-2021, relacionados ao tema. Foram excluídos os trabalhos que não se enquadrassem à metodologia empregada. Resultados: Não há um consenso quanto ao mecanismo biológico envolvido na diabetes e suas implicações à nível ósseo quando se trata de implantes dentários e osseointegração. Sugere-sea realização de maiores pesquisas nessa temática, com um planejamento amostral adequado, para retratar mais fielmente a condição da população com esse perfil. Conclusão: dos 15 artigos selecionados dentre os 318 apresentados inicialmente, 11 trabalhos estabeleceram que o diabetes mellitus não se configura como uma contra indicação absoluta para instalação de implantes dentários, desde que o paciente esteja com seus níveis glicêmicos controlados.

Descritores: Osseointegração; Implantes Dentários; Diabetes Mellitus.

\section{Abstract}

Introduction: Patients with diabetes mellitus have a certain impairment in the healing process, as well as bone neoformation. Objective: To analyze, through a literature review, the feasibility of oral rehabilitation through dental implants in patients diagnosed with Diabetes Mellitus. Material and method: An Systematic literature review was carried out with analysis of articles in the database available at Google Scholar, Lilacs and PubMed. The descriptors "Osseointegration", "Dental Implants" and "Diabetes Mellitus" were used. Texts in any language and sequence according to relevance were selected. The inclusion criteria used were articles and books published between the years 2016-2021, related to the topic. Works that did not fit the methodology used were excluded. Results: There is no consensus on the biological mechanism involved in diabetes and its implications at the bone level when it comes to dental implants and osseointegration. It is suggested to carry out further research on this theme, with an adequate sampling plan, to more faithfully portray the condition of the population with this profile. Conclusion: of the 15 articles selected among the 318 initially presented, 11 studies established that diabetes mellitus is not an absolute contraindication for the installation of dental implants, as long as the patient has controlled blood glucose levels.

Descriptors: Osseointegration; Dental Implants; Diabetes Mellitus.

\section{Resumen}

Introducción: Los pacientes con diabetes mellitus presentan cierta alteración en el proceso de cicatrización, así como neoformación ósea. Objetivo: Analizar, mediante revisión Sistemática de la literatura, la viabilidad de la rehabilitación oral mediante implantes dentales en pacientes diagnosticados de Diabetes Mellitus. Material y método: Se realizó una revisión integradora de la literatura con análisis de artículos en la base de datos disponible en Google Scholar, Lilacs y PubMed. Se utilizaron los descriptores "Osteointegración", "Implantes dentales" y "Diabetes Mellitus". Se seleccionaron textos en cualquier idioma y secuencia según su relevancia. Los criterios de inclusión utilizados fueron artículos y libros publicados entre los años 2016-2021, relacionados con el tema. Se excluyeron los trabajos que no se ajustaban a la metodología utilizada. Resultados: No existe consenso sobre el mecanismo biológico involucrado en la diabetes y sus implicaciones a nivel óseo en cuanto a implantes dentales y osteointegración. Se sugiere realizar más investigaciones sobre este tema, con un adecuado plan de muestreo, para retratar más fielmente la condición de la población con este perfil. Conclusión: de los 15 artículos seleccionados de los 318 presentados inicialmente, 11 estudios establecieron que la diabetes mellitus no es una contraindicación absoluta para la instalación de implantes dentales, siempre y cuando el paciente tenga los niveles de glucosa en sangre controlados.

Descriptores: Oseointegración; Implantes Dentales; Diabetes Mellitus.

INTRODUÇÃO

No atendimento clinico odontológico é imprescindível a realização de uma anamnese detalhada. A partir disso, é possível diagnosticar possíveis fatores considerados de risco para alguns procedimentos odontológicos mais invasivos, como exemplo, o de implantes dentários ${ }^{1}$. A Agência Nacional de Vigilância Sanitária no-466/MS/SVS, datada de 04 de junho de 1998 estabelece que: "paciente de risco é aquele que possui alguma condição potencialmente determinante de sua instabilidade"2. 
A Diabetes Mellitus (DM) é entendida como um transtorno metabólico causando hiperglicemia, ocorrendo em razão a defeitos na secreção ou na ação do hormônio insulina, que é produzido no pâncreas, pelas chamadas células beta. A função principal da insulina é promover a entrada de glicose para as células do organismo de forma que ela possa ser aproveitada para as diversas atividades celulares. A falta da insulina ou um defeito na sua ação resulta em acúmulo de glicose no sangue, o que chamamos de hiperglicemia. Os dois grupos mais comuns do Diabetes são do tipo 1 e do Tipo $2^{3}$.

O Diabetes tipo 1 é autoimune, o qual o sistema imunológico ataca e destrói as células $\beta$ pancreáticas que produzem insulina ${ }^{4}$. $O$ Diabetes tipo 2 ocorre quando há exposição crônica a níveis elevados de glicose e lipídios desencadeando no corpo uma produção prejudicada de insulina pelas células $\beta$ e ainda uma resistência a esse hormônio, ou seja, não reagem à insulina. $O$ diabetes tipo 2 é muito mais comum que o tipo $1^{3,5}$.

A dicotomia de paciente acometido de DM e a reabilitação oral por meio de implantes dentários, esbarram no fato que o DM apresenta dentre vários outros sintomas a cicatrização demorada de feridas ${ }^{6}$. Os principais sintomas dessa doença são: fome e sede excessiva e vontade de urinar várias vezes ao dia. Especificamente, a DM tipo 1: fome frequente, sede constante, vontade de urinar diversas vezes ao dia, perda de peso, fraqueza, fadiga, mudanças de humor, náusea e vômito. Os sintomas do DM tipo 2: fome frequente, Sede constante, formigamento nos pés e mãos, Vontade de urinar diversas vezes, Infecções frequentes na bexiga, rins, pele e infecções de pele, feridas que demoram para cicatrizar, visão embaçada, além das que são evidenciadas na cavidade oralcomo a doença periodontal, cárie dentária, xerostomia, alteração do sabor, hipossalivação e halitose ${ }^{3}$.

Para o sucesso no tratamento com implantes dentários, é de suma importância que aconteça o fenômeno da osseointegração, podendo ser caracterizado como um processo biológico similar ao da cicatrização que consiste na fixação do implante ao tecido ósseo. Este é fundamental para o resultado adequado de implantes dentários e consequentemente, uma melhor qualidade de vida do paciente, devido a melhora tanto na estética quanto na funcionalmente ${ }^{7}$.

Os portadores da diabetes mellitus apresentam certo comprometimento na microcirculação vascular, na atividade imunológica e inflamatória, tornando-os mais vulneráveis ao desenvolvimento de infecções. Esses fatores influenciam negativamente no processo de cicatrização, assim como a neoformação óssea do paciente. Além disso, alta taxa de glicose no sangue interfere no metabolismo ósseo, reduzindo sua densidade mineral, impactando na formação e na qualidade de sua microarquitetura, fatores esses que afetam a osseointegração e 0 sucesso dos implantes ${ }^{8,9}$.

Há evidencias de que a hiperglicemia influencia negativamente na formação óssea, afetando na osseointegração dos implantes dentários. Todavia, a Diabetes Mellitus não configura uma contraindicação absoluta para instalação de implantes dentários, sendo necessário analisar quais as condições que proporcionam indicação segura em pacientes idosos que sejam portadores da $\mathrm{DM}^{10}$.

Este trabalho busca responder se o paciente acometido pela Diabetes Mellitus (DM) poderá ser submetido com a devida segurança a uma reabilitação oral, por meio de implantes dentários. Nessa perspectiva, este estudo analisa a viabilidade da reabilitação oral por meio de implantes dentários em pacientes diagnosticados com Diabetes Mellitus, observando assim, o mecanismo de osseointegração.

MATERIAL E MÉTODO

Este estudo trata-se de uma revisão sistemática da literatura, de caráter qualitativo e quantitativo, utilizando como base livros, revistas científicas e artigos científicos presentes no Google Acadêmico, LILACS Literatura Latino-americana e do Caribe em Ciências da Saúde e PubMed, mecanismo de busca junto ao MEDLINE- Medical Literature Analysis and Retrieval System Online.

Foi realizada a análise de artigos relacionados à temática da pesquisa, buscandose assim fortalecer a construção do referencial teórico. Utilizaram-se os descritores "Osseointegração", "Implantes Dentários" e "Diabetes Mellitus" Foram selecionados textos em "qualquer idioma" e a sequência de acordo com a relevância.

Os critérios de inclusão usados foram artigos, teses, dissertações e livros publicados entre os anos de 2016-2021, em qualquer idioma, que se enquadraram ao tema da pesquisa e que apresentaram metodologia empregada de forma detalhada. Foram excluídos os trabalhos que não se enquadrassem à metodologia empregada, bem como os que não avaliaram o Diabetes Mellitus e suas consequências ao processo de 
cicatrização óssea, bem como, os que não trataram da reabilitação oral por meio de implantes dentários.

Foi realizada a leitura e a seleção dos estudos de acordo com a finalidade de fundamentar um debate acerca da reabilitação oral por meio de implantes dentários em pacientes acometidos pelo Diabetes Mellitus.

\section{RESULTADOS}

Foram extraídos da busca aproximadamente 318 resultados, dos quais foram escolhidos para compor a discussão, 0 número total de 15 estudos, destacados por critérios de relevância científica, sendo que os demais artigos selecionados pelo mesmo critério foram excluídos por terem conclusões semelhantes, sendo assim, esse número apresentou-se suficiente para a elaboração da discussão desse estudo. Visando melhor exemplificar o conteúdo selecionado, elaborouse 0 quadro 1 , no qual os estudos estão catalogados de acordo com o autor/ano, título, tipo de estudo, objetivo e resultados encontrados, de 2016-2021.

Quadro 1. Estudos de acordo com o autor/ano, título, tipo de estudo, objetivo e resultados

\begin{tabular}{|c|c|}
\hline \multicolumn{2}{|c|}{ SILVA et al. (2020) } \\
\hline Título & $\begin{array}{l}\text { Uma revisão literária sobre a influência da diabetes mellitus tipo } 2 \text { no } \\
\text { processo de osseointegração de implantes dentários }\end{array}$ \\
\hline Tipo de Estudo & Revisão de literatura \\
\hline Objetivo & $\begin{array}{l}\text { Revisar a literatura acerca da influência da diabetes mellitus tipo } 2 \text { no } \\
\text { processo de osseointegração de implantes dentários. }\end{array}$ \\
\hline Resultados & $\begin{array}{l}\text { Em suma, os estudos observaram resultados significativos com boas taxas } \\
\text { de sucesso das reabilitaçóes orais com implantes osseointegrados em } \\
\text { pacientes com diabetes mellitus tipo 2, sendo uma alternativa de } \\
\text { tratamento segura, eficaz e viável. }\end{array}$ \\
\hline \multicolumn{2}{|c|}{ MARINHO (2018) } \\
\hline Título & $\begin{array}{l}\text { Avaliação da influência do diabetes mellitus na perda de implantes } \\
\text { dentários }\end{array}$ \\
\hline Tipo de Estudo & Revisão de literatura \\
\hline Objetivo & $\begin{array}{l}\text { Investigar a hipótese se há insucesso com implantes em pacientes } \\
\text { portadores de diabetes mellitus. }\end{array}$ \\
\hline Resultados & $\begin{array}{l}\text { Apesar do pequeno número de pacientes diabéticos da amostra foi possível } \\
\text { observar que houve interferência da doença no índice de sucesso dos } \\
\text { implantes dentários. }\end{array}$ \\
\hline \multicolumn{2}{|c|}{ GUIA et al. (2017) } \\
\hline Título & $\begin{array}{l}\text { A influência da diabetes mellitus tipo } 2 \text { no processo de } \\
\text { osseointegraç âo: umarevisão de literatura }\end{array}$ \\
\hline Tipo de Estudo & Revisão de literatura \\
\hline Objetivo & $\begin{array}{l}\text { Realizar } \\
\text { uma revisão da literatura sobrea influência da diabete tipo } 2 \text { na } \\
\text { osseointegração para,deforma mais efetiva, ter um maior controle das } \\
\text { possíveis complicações e fornecer assim uma melhor assistência ao } \\
\text { paciente. }\end{array}$ \\
\hline Resultados & $\begin{array}{l}\text { Pacientes diagnosticados com diabetes tipo } 2 \text { controlados, apresentam } \\
\text { sucesso na realização de implantes osseointegrados. }\end{array}$ \\
\hline \multicolumn{2}{|l|}{ SILVA (2019) } \\
\hline Título & $\begin{array}{l}\text { As influências de doenças sistêmicas na osseointegração de implantes } \\
\text { dentários }\end{array}$ \\
\hline Tipo de Estudo & Revisão de literatura \\
\hline Objetivo & $\begin{array}{l}\text { Investigar quaisosfatores } \\
\text { /doenças sistêmicas interferem nesse processo de osseointegração } \\
\text { envolvendo implantes dentários. }\end{array}$ \\
\hline Resultados & $\begin{array}{l}\text { Concluiu-se que doenças sistêmicas como Osteoporose, Diabetes Mellitus, } \\
\text { HIV/AIDS, não se configuram por si só razão como contraindicação para a } \\
\text { realização de terapiaodontológica através de implantes, desde que o } \\
\text { paciente esteja compensado por tratamento específico. }\end{array}$ \\
\hline \multicolumn{2}{|l|}{ MOTTA (2019) } \\
\hline Título & $\begin{array}{l}\text { A influência da diabetes mellitus na osseointegraç ão de } \\
\text { implantes dentários. }\end{array}$ \\
\hline Tipo de Estudo & Revisão de literatura \\
\hline Objetivo & Estudar a influência da diabetes no processo de osseointegração. \\
\hline Resultados & $\begin{array}{l}\text { Os resultados encontrados no presente estudo mostraram que a maioria } \\
\text { dos autores demonstrou que o insucesso do implante dentário está } \\
\text { relacionado com o diabetes não controlado. }\end{array}$ \\
\hline \multicolumn{2}{|c|}{ MEDEIROS (2017) } \\
\hline Título & $\begin{array}{l}\text { Osseointegração de implantes dentários em pacientes diabéticos: uma } \\
\text { revisão integrativa daliteratura científica }\end{array}$ \\
\hline Tipo de Estudo & Revisão de literatura \\
\hline Objetivo & $\begin{array}{l}\text { Realizar uma revisão integrativa sobre a relação entre a osseointegração de } \\
\text { implantes dentários e a diabetes. }\end{array}$ \\
\hline Resultados & $\begin{array}{l}\text { Concluiu-se com o estudo que quando o paciente diabético está } \\
\text { compensado, ou seja, controlado sistemicamente, esta condição não } \\
\text { interfere na osseointegração dos implantes dentários. }\end{array}$ \\
\hline
\end{tabular}

Quadro 1 (continuação). Estudos de acordo com o autor/ano, título, tipo de estudo, objetivo e resultados

\begin{tabular}{|c|c|}
\hline \multicolumn{2}{|c|}{ SANTOS et al. (2018) } \\
\hline Título & Diabete melito tipo 2 e osseointegração: revisão de literatura \\
\hline Tipo de Estudo & Revisão de literatura \\
\hline Objetivo & $\begin{array}{l}\text { Relatar a influência do diabete melito tipo } 2 \text { no processo de } \\
\text { osseointegracão de implantes dentários. }\end{array}$ \\
\hline Resultados & $\begin{array}{l}\text { O DM2 influencia o processo de cicatrização óssea, sua modulação, } \\
\text { dimimuindo a mineralização, o contato osso/ implante, aumentando } \\
\text { reação inflamatória, imunológica,comconsequências para } \\
\text { osseointegração, comprometendo assim a estabilidade, sucesso e } \\
\text { sobrevida dos implantes. }\end{array}$ \\
\hline \multicolumn{2}{|r|}{  } \\
\hline Título & $\begin{array}{l}\text { Interferência do diabetes mellitus no processo de osseointegração do } \\
\text { implante dentário }\end{array}$ \\
\hline Tipo de Estudo & Revisão de literatura \\
\hline Objetivo & $\begin{array}{l}\text { Pesquisar através da revisão literária, a influência do diabetes } \\
\text { mellitus (DM) sobre o processo de osseointegração de implanteS } \\
\text { dentários em pacientes diabéticos; verificando as indicações e } \\
\text { contraindicações clínicas }\end{array}$ \\
\hline Resultados & $\begin{array}{l}\text { Foi visto nesta revisão que o DM não se configura como uma } \\
\text { contraindicação absoluta para a instalação de implantes dentários, } \\
\text { sendo importante que o paciente, ao ser implantado com esta } \\
\text { patologia, esteja com a mesma controlada, especialmente durante o } \\
\text { período de osseointegração. }\end{array}$ \\
\hline \multicolumn{2}{|c|}{ VALLE et al. (2017) } \\
\hline Título & Influência do Diabetes Mellitus sobre os tecidos periimplantares \\
\hline Tipo de & \\
\hline Objet & $\begin{array}{l}\text { osseointegração de acordo com } \\
\text { s. }\end{array}$ \\
\hline Resultados & $\begin{array}{l}\text { A presença do diabetes quando controlado não contra indica a } \\
\text { terapia de implante, ao contrário: permite boas taxas de } \\
\text { sobrevivência e previsibilidade no tratamento }\end{array}$ \\
\hline \multicolumn{2}{|c|}{ PINHEIRO (2019) } \\
\hline Título & o de Implantes em \\
\hline Tipo de Es & Revisão de liter \\
\hline Objetivo & $\begin{array}{l}\text { Fazer uma avaliação histomorfométrica da osseointegração de } \\
\text { implantes dentários nesses pacientes, respondendo ao } \\
\text { questionamento se a quantidade de tecido ósseo formado ao redor } \\
\text { dos implantes dentários é semelhante entre pacientes com síndrome } \\
\text { metabólica e pacientes saudáveis. }\end{array}$ \\
\hline Resultados & $\begin{array}{l}\text { não ocorreu diferença significativa } \\
\text { relação a quantidade de tecido ósseo }\end{array}$ \\
\hline \multicolumn{2}{|l|}{ VALE (2018) } \\
\hline Título & $\begin{array}{l}\text { Efeito dc } \\
\text { review }\end{array}$ \\
\hline & literatura \\
\hline Objetivo & $\begin{array}{l}\text { ma "umbrella review" sobre o efeito do diabetes mellitus } \\
\text { tes dentários. }\end{array}$ \\
\hline Resultados & $\begin{array}{l}\text { Concluiu-se que pacientes diabéticos, desde que controlados, } \\
\text { apresentam risco igual de falha, mucosite e periimplantite à } \\
\text { pacientes não diabéticos, mas maior risco à periimplantite quando } \\
\text { não controlados. }\end{array}$ \\
\hline \multicolumn{2}{|l|}{ E (20) } \\
\hline Título & $\begin{array}{l}\text { Diabetes mellitus e sua correlação com a implantodontia: uma } \\
\text { revisão da literatura }\end{array}$ \\
\hline & \\
\hline Objetiv & $\begin{array}{l}\text { ado quadro que a Diabetes Mellitus } \\
\text { em implantodontia. }\end{array}$ \\
\hline Resu & $\begin{array}{l}\text { O trabalho pôde mostrar que a dualidade diabetes e odontologia } \\
\text { fazem parte de um complexo universo e que este quadro necessita de } \\
\text { uma visão mais aguçada, pois compreende uma série de } \\
\text { complicações se tratada de maneira inadequada. }\end{array}$ \\
\hline \multicolumn{2}{|l|}{ SANTOS (202 } \\
\hline Título & $\begin{array}{l}\text { Impacto da Diabetes Mellitus tipo } 2 \text { descompensa da na } \\
\text { osseointegração de implante dentário. }\end{array}$ \\
\hline I1po & \\
\hline Objeti & $\begin{array}{l}\text { da diabetes tipo } 2 \\
\text { dentário. }\end{array}$ \\
\hline Resulta & $\begin{array}{l}\text { A Diabetes Mellitus Tipo II descompensada intervém no processo } \\
\text { cicatrização ósseo, remodelação do mesmo, mineralização e retardo } \\
\text { no processo de cicatrização tecidual, aumentando o risco a infecções } \\
\text { inflamatórias, comprometendo assim a sobrevida e estabilidade do } \\
\text { implante. A A relação da osseointegração e a diabetes mellitus é } \\
\text { favorável desde que os níveis de glicose no sangue estejam } \\
\text { corretamente controlados durante o procedimento e no período de } \\
\text { osseointegração. - Não há contra indicação absoluta para o } \\
\text { tratamento de implante dentário em pacientes diabéticos, desde que } \\
\text { os níveis de glicose estejam controlados }\end{array}$ \\
\hline \multicolumn{2}{|l|}{ VARE. } \\
\hline Título & $\begin{array}{l}\text { Reabilitação através de implantes dentários no paciente portador de } \\
\text { diabete melitus: revisão de literatura }\end{array}$ \\
\hline Tipo de E & Revisão de literatura \\
\hline Objetivo & $\begin{array}{l}\text { Analisar os protocolos clínicos a serem seguidos nos tratamentos } \\
\text { com implantes dentários em pacientes portadores de Diabete } \\
\text { Melitus. }\end{array}$ \\
\hline Resultados & $\begin{array}{l}\text { Concluiu-se que o paciente diabético, desde que esteja com os níveis } \\
\text { glicêmicos controlados, pode ser submetido a tratamento com } \\
\text { implantes osseointegrados. }\end{array}$ \\
\hline
\end{tabular}
Fonte: dados da pesquisa

DISCUSSÃO

O tratamento com implantes na Odontologia como procedimento reabilitador tem seus desafios, pois traz consigo as necessidades do paciente, embora as complicações possam estar presentes. Devolver função, autoestima, estética, fonética e saúde, 
são os maiores objetivos na implantodontia ${ }^{10,15,24}$. Porém, como em todo procedimento cirúrgico, riscos e falhas estão susceptíveis a ocorrer, por isso é necessários alguns cuidados para que não ocorram. Sabendo que na bibliografia estudada não há previsibilidade de quanto tempo o paciente precisa-se manter a Diabetes compensada para a realização da cirurgia de implantes dentários, deve sim, ser considerado conforme estabelece a American Society of Anesthesiologists (ASA), ASA I ou II, pois a análise das patologias como o diabetes correlaciona-se com o risco cirúrgico: quanto mais severa a disfunção sistêmica, maior o risco do ato operatório. Na maioria das vezes, os pacientes geriátricos atendidos na clínica odontológica podem ser enquadrados na seguinte classificação: ASA II (doença sistêmica de leve a moderada) - raramente os idosos não têm alguma alteração sistêmica (ASA I), condição ideal à intervenção cirúrgica ${ }^{4,10,21,25}$.

Silva et al. ${ }^{11}$ concluiram que três estudos relataram diferenças no sucesso clínico de implantes inseridos em pacientes diabéticos e não diabéticos. Modificações no processo de cicatrização após a cirurgia foram observadas em dois desses estudos. Em um dos estudos, os parâmetros inflamatórios periodontais e periimplantares foram piores em pacientes com prédiabetes e com diabetes mellitus tipo $2 \mathrm{em}$ comparação com controles saudáveis ${ }^{11}$.

Marinho $^{12}$ em seu estudo sobre a avaliação da influência do diabetes mellitus na perda de implantes dentários identificou que de acordo com a metodologia empregada, pode-se afirmar que há a interferência do diabetes mellitus nos índices de sucesso dos implantes dentários osseointegrados e que o controle da co-morbidade é essencial para redução dos insucessos e obtenção de uma boa resposta clínica.

Guia et al. ${ }^{13}$ afirmaram que diante da revisão bibliográfica a literatura pontua que pacientes diagnosticados com diabetes tipo 2 controlados, apresentam sucesso na realização de implantes osseointegrados. Neste estudo os autores concluíram que o controle glicêmico pré e pós-operatório deve ser obtido para uma melhor osseointegração e sucesso no implante.

Sobre a osseointegração de implantes dentários em pacientes diabéticos com uma revisão integrativa de literatura cientifica, Medeiros $^{15}$ (2017) conclui ser controverso o mecanismo biológico envolvido na diabetes que gera alterações principalmente à nível ósseo quando falamos de implantes dentários e osseointegração, devendo-se promover mais pesquisas nessa temática, com maior número amostral, o que irá retratar mais fielmente a condição da população com esse perfil ${ }^{15,19}$.

CONCLUSÃO

De acordo com a revisão realizada neste trabalho, conclui-se que a Diabetes Mellitus não se caracteriza como uma contraindicação absoluta para instalação de implantes dentários, desde que os níveis glicêmicos do paciente estejam devidamente controlados, é importante ressaltar sobre a realização de uma apurada anamnese pelo cirurgião-dentista afim de que o mesmo saiba conduzir o melhor tratamento reabilitador com implante dentário.

\section{REFERÊNCIAS}

1. Motta AKS. A Influência da Diabetes Mellitus na Osseointegração de Implantes Dentários [monografia]. São Paulo: Faculdade Sete Lagoas - FACSETE; 2019.

2. Agencia Nacional de Vigilância Sanitária ANVISA. Portaria da Agência Nacional de Vigilância Sanitária nำ66/MS/SVS. Brasília, DF: ANVISA, 1998.

3. Brasil. Ministério da Saúde. Secretaria de Atenção à Saúde. Departamento de Atenção Básica. Estratégias para o cuidado da pessoa com doença crônica: Diabetes Mellitus. Brasília, DF, 2013.

4. Katsarou A, Gudbjörnsdottir S, Rawshani A, Dabelea D, Bonifacio E, Barbara J, et al. Type 1 diabetes mellitus. Nat Rev Dis Primers. 2017, 17016.

5. Rehma K, Akash, MSH. Mechanism of generation of oxidative stress and pathophysiology of type 2 diabetes mellitus: how are they interlinked? J cell biochem. 2017; 118:3577-85,

6. Jardim JCM, Cortelli JR. Avaliação dos mecanismos de cicatrização de feridas periodontais em pacientes diabéticos e não diabéticos. Braz J Periodontol. 2019; 29.

7. Mendes VC, Davies JE. Uma nova perspectiva sobre a biologia da osseointegração. Rev Assoc Paul Cir Dent. 2016; 70:166-71.

8. Diniz DR. Osseointegração em pacientes diabéticos [monografia]. Pindamonhangaba: Faculdade de Pindamonhangaba; 2016.

9. Martins V, Bonilha T, Falcón-Antenucci RM, Verri AC, Verri FR. Osseointegração: análise de fatores clínicos de sucesso e insucesso. Rev Odontol Araçatuba. 2011;32:26-31.

10. Pereirinha CIS. Influência da Diabetes Mellitus na osteointegração [dissertação]. Porto (Portugal): Universidade Fernando Pessoa; 2011.

11. Silva ADF, Rodrigues ALLV, Araújo MA, Maciel FWH, de Macedo ACT, Avelar RL et al. Uma revisão literária sobre a influência da diabetes mellitus tipo 2 no processo de 
osseointegração de implantes dentários. Braz J Hea Rev. 2020; 3:11277-292.

12. Marinho LMRF. Avaliação da influência do diabetes mellitus na perda de implantes dentários [dissertação]. Piracicaba: Faculdade de Odontologia de Piracicaba da Universidade Estadual de Campinas; 2018.

13. Guia EBS, Silva SM, Gatis MCQ. A influência da diabetes mellitus tipo II no processo de osseointegração: uma revisão de literatura. Cadernos de Graduação. 2017; 3:75-82.

14. Silva WLRX. As influências de doenças sistêmicas na osseointegração de implantes dentários [monografia] Natal: Faculdade de Odontologia, Universidade Federal do Rio Grande do Norte; 2019

15. Medeiros FLA. Osseointegração de implantes dentários em pacientes diabéticos: uma revisão integrativa da literatura científica [monografia]. Natal: Departamento de Odontologia da Universidade Federal do Rio Grande do Norte; 2017.

16. Santos RC, Pinho RCM, Cimões R. Diabete melito tipo 2 e osseointegração: revisão de literatura. Periodontia. 2018;28(4):36-40.

17. Silva TAV. Interferência do diabetes mellitus no processo de osseointegração do implante dentário [monografia]. Recife: Faculdade Sete Lagoas FACSETE; 2016.

18. Valle LSEMB, Oliveira PC, Silva LF, Ramires GADA, Souza FA, Garcia Junior IR, Bassi APF, Ponzoni D. Influência do Diabetes Mellitus sobre os tecidos periimplantares. Arch Health Invest. 2017;6(Spec Iss 2):258.

19. Pinheiro FP. Avaliação histomorfométrica da osseointegração de implantes em pacientes com alterações metabólicas [dissertação] Duque de Caxias: Universidade do Grande Rio "Professor José de Souza Herdy'; 2019.

20. Vale DA. Efeito do diabetes mellitus sobre os implantes dentários: umbrella review dissertação. São Paulo: Universidade Ibirapuera; 2018.

21. Almeide LJS. Diabetes mellitus e sua correlação com a implantodontia: uma revisão da literatura [monografia]. Sete Lagoas: Faculdade Sete Lagoas FACSETE; 2017.

22. Santos DC. Impacto da Diabetes Mellitus tipo II descompensada na osseointegração de implante dentário [monografia]: Taubate: Unitau; 2020

23. Varejão GZM. Reabilitação através de implantes dentários no paciente portador de diabete melitus: revisão de literatura [monografia]. Vitória: Faculdade Sete lagoas FACSETE. Vitória; 2016

24. Carvalho PSP, Pellizzer EP. Fundamentos em Implantodontia: Uma visão contemporânea. São Paulo: Quintessence; 2011.
25. International Diabetes Federation. IDF Diabetes Atlas: $8^{\text {th }}$ 2017. International Diabetes Federation; 2017.

\section{CONFLITO DE INTERESSES}

Os autores declaram não haver conflitos de interesse

AUTOR PARA CORRESPONDÊNCIA

Herrison Félix Valeriano da Silva

Universidade Federal da Paraíba (UFPB)

58051-900 João Pessoa - PB, Brasil

E-mail: herrison.felix.vds@gmail.com
Submetido em 25/07/2021

Aceito em 05/01/2022 